\title{
FIELD-DRIVEN BROWNIAN MOTION OF MAGNETIC DOMAIN WALLS
}

\author{
Lukáš Glod ${ }^{*}$ _ Gabriela Vasziová ${ }^{* *}$ \\ Jana Tóthová ${ }^{* *}$ — Vladimír Lisý ${ }^{* *}$
}

\begin{abstract}
The dynamics of a magnetic domain wall (DW) in a wire is studied. The DW is modeled as a Brownian particle subjected to thermal fluctuations and is characterized by the mass, position and velocity. Its motion is damped by friction, pinned by the irregularities in the material and driven by a constant force due to the external magnetic field. We have obtained the corresponding Langevin equation that contains a white-noise force. The use of an effective method taken from the statistical physics allowed us to convert this stochastic equation into an ordinary differential equation. From its solution the mean square displacement of the DW with other relevant time correlation functions and their spectral densities have been found. The electric current induced by the moving DW is also calculated.
\end{abstract}

K e y w ords: magnetic domain wall, motion in wires, thermal fluctuations, Brownian motion, induced electric current

\section{INTRODUCTION}

Magnetic domain walls (DW) are topological objects that appear in magnets as localized changes in the magnetization. They attract a great interest due to the variety of interesting properties and also because of important applications, for example in high-density memory storages [1]. DWs can be manipulated by electric and magnetic fields. Recently [2], using an alternating electric current, a resonance motion of the DW has been induced in a ferromagnetic nanowire. Considering the DW as a particle-like excitation [3], its motion was described as small oscillations of a harmonic oscillator under an external force. Detecting the resonance, the mass of such a "particle" $\left(6.6 \times 10^{-23} \mathrm{~kg}\right)$ has been determined. Due to the law mass of the DW, one could expect that thermal fluctuations play an important role in the DW motion. Such fluctuations were however not accounted for in the work [2]. This is the main aim of the present work. Although the DWs are complex objects that are difficult to treat theoretically, for small chiralities it is possible to describe rigid DWs by linear equations for its position and chirality [4]. In the present contribution we use the model for the rigid DW as a particle and study its motion in a wire. The particle is subject to thermal fluctuations and dissipates the energy due to the friction. In addition, it is driven by a constant force arising from the external magnetic field. We thus have a deterministic motion superimposed by the chaotic one, as it is in the case of a real Brownian particle (BP). With this representation, we can apply a number of results and methods developed within the statistical mechanics [5]. We take advantage of this and use an effective but little known method that allows us converting the stochastic Langevin equation, derived for the DW, to a deterministic equation for the DW mean square displacement (MSD). The solution of this equation is used to find also other relevant time correlation functions for the DW, such as its velocity autocorrelation function (VAF) or the time dependent diffusion coefficient. Their spectral densities are also calculated. The moving DW induces a detectable current. Although we calculate this current coming from the recent theory by Lucassen and Duine [4], our results notably differ from the calculations by these authors. So, in their work the mean position of the DW and the resulting electric current do not depend on the time. However, if the wire is placed in a constant magnetic field, the studied problem is equivalent to that of a one-dimensional motion of a confined BP driven by an external force. The motion of the DW can be then considered as a motion of a particle in a potential well. The present contribution suggests a simple solution of this task. We have shown that due to the constant force acting on the DW its mean position is nonzero and changes with the time together with the current. We have found the full solution of this problem. The obtained solution is applied to finding the mean electric current induced by a field-driven DW. The results are illustrated by numerical calculations using the DW parameters corresponding to the experiment [2].

\section{DOMAIN WALL FLUCTUATIONS AND THE INDUCED CURRENT}

The current induced by the DW motion is given by the expression

$$
I(t) \sim \dot{\phi}(t)-\frac{\beta}{\lambda} \dot{x}(t)
$$

\footnotetext{
* Department of Mathematics and Physics, Institute of Humanitarian and Technological Sciences, The University of Security Management, Kukučínova 17, 04001 Košice, Slovakia, lukas.glod@vsbm.sk

** Department of Physics, Faculty of Electrotechnics and Informatics, Technical University of Košice, Park Komenského 2,04200 Košice, Slovakia, jana.tothova@tuke.sk, vladimir.lisy@tuke.sk, gabriela.vasziova@tuke.sk
} 


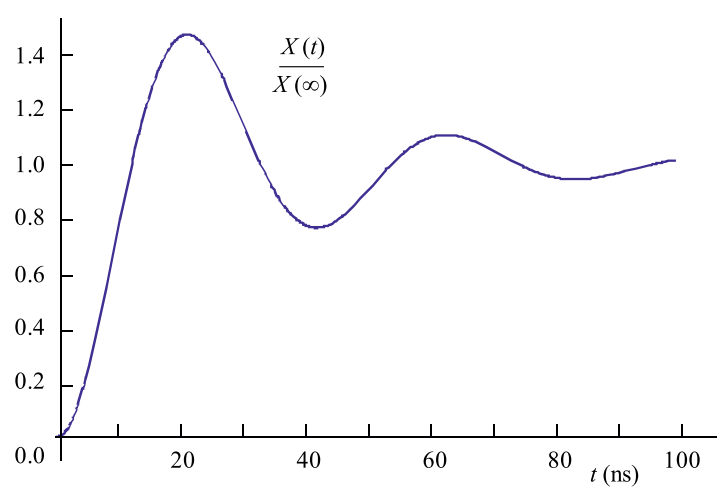

Fig. 1. Normalized MSD from (7) of the DW oscillating with respect to its average position. The parameters are from [2], see the text.

where $\phi, x$ and $\lambda$ are the DW chirality, position and width, respectively, and $\beta$ is a phenomenological parameter [4]. For the derivation of this expression and the proportionality constant $C$ in (1) see $[6,7]$. Up to the linear order in time derivatives $C$ depends on the conductivities of the majority and minority electrons, the length and cross-sectional area of the sample. In (1), the quantities $x$ and $\phi$ are of stochastic nature. They obey the system of equations for the DW in a parabolic potential. The linearized $(\phi \rightarrow 0)$ form of these equations is

$$
\begin{aligned}
& \frac{\dot{x}}{\lambda}=\alpha \dot{\phi}+2 \frac{K}{\hbar} \phi+\sqrt{\frac{D}{2}} \eta_{1}(t), \\
& \dot{\phi}=-\alpha \frac{\dot{x}}{\lambda}-2 \omega_{\text {pin }} \frac{x}{\lambda}+\sqrt{\frac{D}{2}} \eta_{2}(t) .
\end{aligned}
$$

Here $\alpha$ denotes the Gilbert damping and $K$ is the transverse-magnetic-anisotropy energy. It is assumed that the pinning force $F_{\text {pin }}$ that accounts for the irregularities in the material depends only on the DW position and is described by the potential that is quadratic in $x$. As a consequence of the fluctuation-dissipation theorem [8], the stochastic forces $\eta_{i}$ with zero mean that describe the thermal fluctuations are at different moments of time statistically independent, $\left\langle\eta_{i}(t) \eta_{j}\left(t^{\prime}\right)\right\rangle=$ $\delta_{i j} \delta\left(t-t^{\prime}\right)$, and their intensity is determined by the constant $D=4 \alpha k_{B} T / \hbar N_{w}$, here we have corrected the numerical coefficient 2 (as given in [4]) to 4 , and $k_{B}$ is the Boltzmann constant, $T$ is the temperature, and $N_{w}$ is the number of spins in the DW [4].

If the random forces are not considered, from (2) and (3) the deterministic equations for the motion of DW can be derived. The equation for the DW coordinate,

$$
\left(1+\alpha^{2}\right) \frac{\ddot{x}}{\lambda}+2 \alpha\left(\omega_{\text {pin }}+\frac{K}{\hbar}\right) \frac{\dot{x}}{\lambda}+4 \omega_{\text {pin }} \frac{K}{\hbar} \frac{x}{\lambda}=0
$$

corresponds to the equation of motion for a noisy harmonic oscillator [9],

$$
m \ddot{x}+\gamma \dot{x}+m \omega_{o}^{2} x=f(t)
$$

where the influence of the thermal fluctuations has been already accounted for by the Langevin force $f(t)$ [10]. The mass of the DW is $m=\hbar^{2} N_{w} / K \lambda^{2}$ [2], $\gamma$ is the friction factor and $\omega_{0}$ the oscillator frequency.

According to the Wiener-Khinchin theorem, the spectrum (more exactly, the spectral density) of the fluctuating current is determined from the time correlation function $\left\langle I(t) I\left(t^{\prime}\right)\right\rangle$ as its Fourier transform [8,11]. If (1) is inserted in this correlator with the use of $\dot{\phi}(t)$ from (3), one has to average the products $x(t) x\left(t^{\prime}\right), \dot{x}(t) \dot{x}\left(t^{\prime}\right)$, $\dot{x}(t) x\left(t^{\prime}\right)$, and $x(t) \dot{x}\left(t^{\prime}\right)$. Besides them, there will be products of the quantities $x$ and $\dot{x}$ with the random force $\eta$ and the term $\sim \eta(t) \eta\left(t^{\prime}\right)$, which, after the averaging, yields the delta function $\delta\left(t-t^{\prime}\right)$. The former products can be omitted since, due to very different time scales characterizing the changes of $x(t), \dot{x}(t)$ and $\eta(t)$, their statistical averages can be put to zero. We thus have to average

$$
\begin{gathered}
I(t) I\left(t^{\prime}\right) \sim\left(\frac{\alpha+\beta}{\lambda}\right)^{2} \dot{x}(t) \dot{x}\left(t^{\prime}\right)+\left(\frac{2 \omega_{\text {pin }}}{\lambda}\right)^{2} x(t) \dot{x}\left(t^{\prime}\right) \\
2 \omega_{\text {pin }} \frac{\alpha+\beta}{\lambda^{2}}\left[\dot{x}(t) x\left(t^{\prime}\right)+x(t) \dot{x}\left(t^{\prime}\right)\right]+\frac{D}{2} \eta(t) \eta_{2}\left(t^{\prime}\right) .
\end{gathered}
$$

To do this, one can use the solution of the corresponding Fokker-Planck equation for the probability density of the quantities $x$ and $\dot{x}$ [12]. Here we apply a different very efficient method. Let us multiply (4a) (with the Langevin force on the right hand side) by $x(t)$, then use the identity $x \ddot{x}=\mathrm{d}(x \dot{x}) / \mathrm{d} t-\dot{x}^{2}$, the statistical independence of $x$, $\dot{x}$ and $f$, and the equipartition theorem $m\left\langle\dot{x}^{2}\right\rangle=k_{B} T$. Equation (4a) can be then rewritten as

$$
m \frac{\mathrm{d}^{2}}{\mathrm{~d} t^{2}}\left\langle x^{2}\right\rangle+\gamma \frac{\mathrm{d}}{\mathrm{d} t}\left\langle x^{2}\right\rangle+2 m \omega_{0}^{2}\left\langle x^{2}\right\rangle=2 k_{B} T .
$$

If we now subtract the equation that follows from (4a) for the correlator $\langle x(t) x(0)\rangle$, we obtain

$$
\begin{array}{r}
m \ddot{X}(t)+\gamma \dot{X}(t)+m \omega_{0}^{2} X(t)+m \omega_{0}^{2}\left(\left\langle x^{2}(t)\right\rangle-\left\langle x^{2}(0)\right\rangle\right) \\
=2 k_{B} T
\end{array}
$$

where $X(t)=\left\langle\Delta x^{2}(t)\right\rangle=\left\langle[x(t)-x(0)]^{2}\right\rangle$ is the MSD of the oscillator. For stationary random processes the last term on the left hand side of (4c) is equal to zero. We thus come to the Vladimirsky rule formulated long ago for more general conditions, including non-Markovian processes (the only restriction is the linearity of the process) [13]. The evident initial conditions for the ordinary differential equation $(4 \mathrm{c})$ are $X(0)=\dot{X}(0)=0$. The solution of the final equation

$$
m \ddot{X}+\gamma \dot{X}+m \omega_{0}^{2} X=2 k_{B} T
$$

for the conditions of the experiment [2] (when 1 $\left.\left(2 m \omega_{0} / \gamma\right)^{2}<0\right)$, is for $t>0$

$$
X(t)=\frac{2 k_{B} T}{m \omega_{0}^{2}}\left[1-\exp \left(-\frac{\gamma}{2 m} t\right)\left(\cos \omega_{1} t+\frac{\gamma}{2 m \omega_{1}} \sin \omega_{1} t\right)\right],
$$




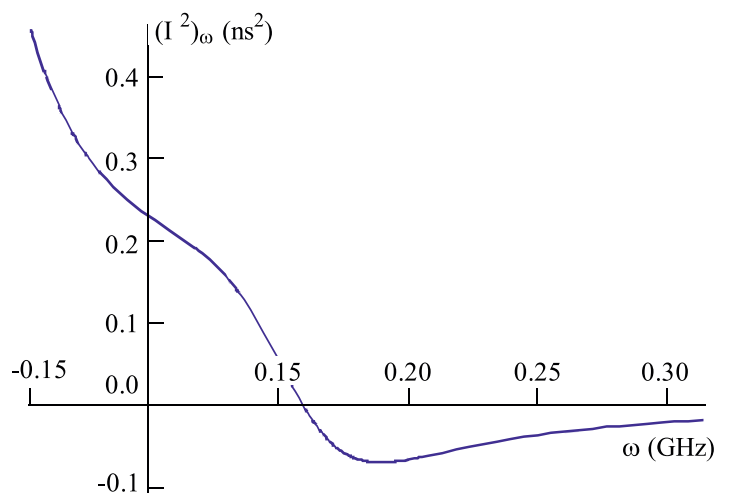

Fig. 2. The dependence of $\left(I^{2}\right)_{\omega}$ on the frequency $\omega$ at $\beta=0$. For the normalization see the text.

where $\omega_{1}=\left[\omega_{0}^{2}-(\gamma / 2 m)^{2}\right]^{1 / 2}$. The corresponding numerical calculation is shown in Fig. 1. The parameters used in the calculations are determined from the experiment [2]: $\gamma / m=1 / \tau=0.714 \times 10^{8} \mathrm{~s}^{-1}(\tau$ is the relaxational time of the DW), $\lambda=7 \times 10^{-8} \mathrm{~m}, K=1.8 \times 10^{-24} \mathrm{~J}$, and $\omega_{0}=1.57 \times 10^{8} \mathrm{~s}^{-1}$. For the limit $X(\infty)=2 k_{B} T / m \omega_{0}^{2}$ we have, $e g$ at $T=300 \mathrm{~K}, X(\infty)=5.1 \times 10^{-15} \mathrm{~m}^{2}$.

\section{SPECTRAL DENSITY OF THE INDUCED CURRENT}

Using (7), other time correlation functions can be easily found, such as the VAF, which is not directly measured but is often searched for in computer simulations,

$$
\langle v(t) v(0)\rangle=\frac{1}{2} \ddot{X}(t)
$$

or the time-dependent diffusion coefficient $D(t)=\dot{X}(t) / 2$. The measurable quantity is also the spectral density of the current, $\left(I^{2}\right)_{\omega}$. If we define the Fourier transformation of $I(t)$ as

$$
I_{\omega}=\frac{1}{2 \pi} \int_{-\infty}^{\infty} I(t) \exp (i \omega t) \mathrm{d} t
$$

then, for the stationary random process $[8]$

$$
\left(I^{2}\right)_{\omega}=\frac{1}{\pi}\langle I(t) I(0)\rangle \cos (\omega t) \mathrm{d} t .
$$

It can be found without calculating the correlation functions (combinations of the velocity $v=\dot{x}$ and position $x$ of DW) in $\langle I(t) I(0)\rangle$. This is because the spectral density of the current, $\left(I^{2}\right)_{\omega}$, can be expressed through the densities $\left(v^{2}\right)_{\omega},\left(x^{2}\right)_{\omega}=\omega^{-2}\left(v^{2}\right)_{\omega},(v x)_{\omega}=-i \omega^{-1}\left(v^{2}\right)_{\omega}$, and $(x v)_{\omega}=i \omega^{-1}\left(v^{2}\right)_{\omega}$, that correspond to the correlation functions $\langle\dot{x}(t) \dot{x}(0)\rangle,\langle x(t) x(0)\rangle,\langle\dot{x}(t) x(0)\rangle$, and $\langle x(t) \dot{x}(0)\rangle$, respectively. Then

$$
\left(I^{2}\right)_{\omega} \sim \frac{1}{\lambda^{2}}\left[(\alpha+\beta)^{2}+\left(\frac{2 \omega_{\text {pin }}}{\omega}\right)^{2}\right]\left(v^{2}\right)_{\omega}+\frac{D}{4 \pi} .
$$

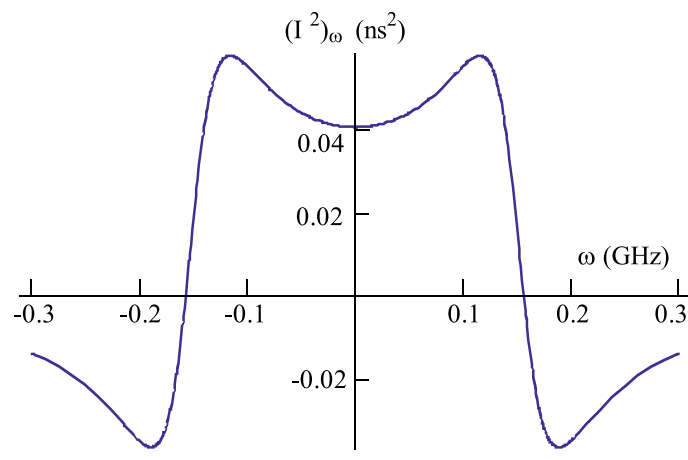

Fig. 3. The same as in Fig. 2 but without extrinsic pinning

requires only the spectrum $\left(v^{2}\right)_{\omega}$,

$$
\left(v^{2}\right)_{\omega}=\frac{1}{\pi} \int_{0}^{\infty}\langle\dot{x}(t) \dot{x}(0)\rangle \cos (\omega t) \mathrm{d} t .
$$

For the even extension of the VAF to $t<0$ it is found from (7) and (8) in the compact form

$$
\left(v^{2}\right)_{\omega}=\frac{k_{B} T}{\pi m} \frac{\omega_{0}^{2}-\omega^{2}}{\left(\omega_{0}^{2}-\omega^{2}\right)^{2}+(\gamma \omega / m)^{2}} .
$$

After its substitution in (11) we directly obtain the spectrum of the current induced by the moving DW. This spectrum does not depend on whether the DW oscillates in the harmonic potential or its MSD only monotonically approaches the value $2 k_{B} T /\left(m \omega_{0}^{2}\right)$. At the frequency $\omega_{0}$ the spectral density crosses the value $D / 4 \pi \sim T$, which could be examined experimentally. Note that the obtained result significantly differs from that in [4], found by a different method. As distinct from the approach used in [4], our method is much simpler and allows for easy proving.

Finally, let us express the quantities entering the velocity spectrum (13) through the DW parameters. These parameters are obtained by comparison of the equation for the DW position (4) and the equation for the harmonic oscillator (4a). The mass of the DW is given above after $(4 \mathrm{a})$, and the friction constant and the oscillator frequency are

$$
\frac{\gamma}{m}=\frac{2 \alpha}{1+\alpha^{2}}\left(\omega_{\text {pin }}+\frac{K}{\hbar}\right), \quad \omega_{0}^{2}=\frac{4 \omega_{\text {pin }}}{1+\alpha^{2}} \frac{K}{\hbar} .
$$

As to the parameters $\alpha$ and $\beta$, in the previous calculations [4] $\alpha$ from 0.01 to 0.1 and $\beta / \alpha=0,1$, and 2 were used as the values for typical materials. However, it follows from the experiment [2] that $\alpha$ should be much smaller. Indeed, for small $\alpha$ we have from (14) $\omega_{\text {pin }} \approx \gamma /(2 \alpha m)-K / \hbar$. To have the pinning potential positive, $\alpha$ should obey the inequality $\alpha<\gamma \hbar /(2 m K)$, which is a value about 0.002 or smaller. We thus can use $\omega_{0}^{2} \approx 4 \omega_{\text {pin }} K / \hbar$. Then $\omega_{\text {pin }} \approx 0.36 \times 10^{6} s^{-1} \approx$ $0.2 \times 10^{-4} K / \hbar$ (the value lower than in [4]) and in the calculations we take $\alpha \approx 0.002$. The results for $\left(I^{2}\right)_{\omega}$ are 


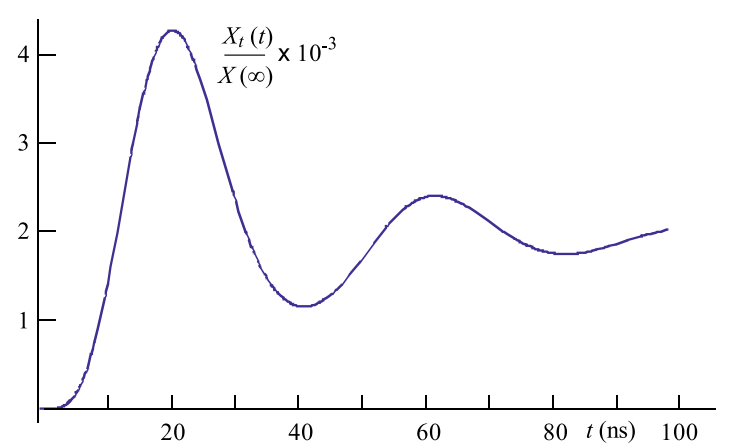

Fig. 4. Normalized MSD of the DW from (22). The parameters are from [2] with the external magnetic field $B$ as described in the text.

presented in Figs. 2 and 3. The spectrum is normalized to $C^{2}$, then shifted down by a constant $D / 4 \pi$ and divided by $10^{9} k_{B} T(\alpha+\beta)^{2} /\left(\pi m \lambda^{2}\right)$. Figure 2 demonstrates the spectrum for nonzero pinning potential. Figure 3 shows the spectrum at $\omega_{\text {pin }}=0$, when it is independent on $\beta$. In all the cases the dependence of $\left(I^{2}\right)_{\omega}$ on $\omega$ is very different from the calculations in [4].

\section{FIELD-DRIVEN MOTION OF DOMAIN WALLS}

Let us now consider the case when the wire is placed in an external magnetic field. Then, if the field is parallel to the easy axis of the sample ( $z$ direction), (3) must be changed to

$$
\dot{\phi}=-\alpha \frac{\dot{x}_{t}}{\lambda}-2 \omega_{\text {pin }} \frac{x_{t}}{\lambda}+\frac{g \mu_{B} B}{\hbar}+\sqrt{\frac{D}{2}} \eta_{2}(t),
$$

$i e$, now it contains a term proportional to the field $g B$. As distinct from the work [4], we take into account the pinning. Combining Eqs. (2) and (15), instead of (4a) the following equation of motion can be obtained for the DW having the position $x_{t}$ in the laboratory system

$$
m \ddot{x}_{t}+\gamma \dot{x}_{t}+m \omega_{0}^{2} x_{t}=F+f(t) .
$$

Here the new force $F$ has been introduced,

$$
F=\frac{2 N_{w}}{1+\alpha^{2}} \frac{g \mu_{B} B}{\lambda}
$$

Let us denote as $x^{*}$ the position of the DW with respect to the frame where the motion is determined by (16) without the random force $f$. The quantity $x^{*}$ is the solution of the deterministic equation

$$
m \ddot{x}^{*}+\gamma \dot{x}^{*}+m \omega_{0}^{2} x^{*}=F .
$$

For the difference $x(t)=x_{t}(t)-x^{*}(t)$ we have the equation

$$
m \ddot{x}+\gamma \dot{x}+m \omega_{0}^{2} x=f(t) .
$$

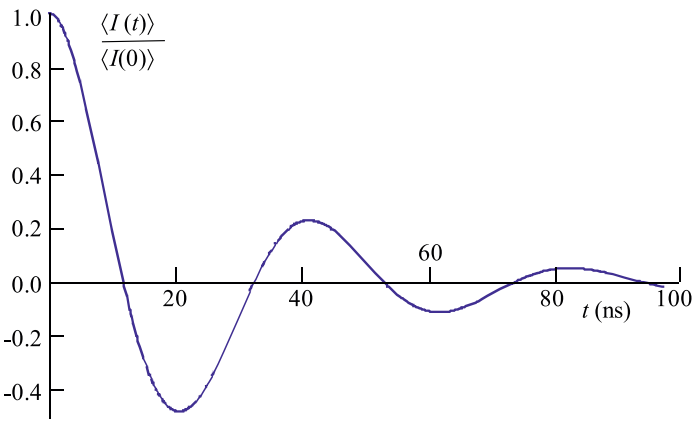

Fig. 5. Normalized mean current induced by the moving DW, from $(24)$

Instead of this stochastic equation we can immediately write (6) for the MSD of the DW position with respect to $x^{*}$. It should be again solved with the conditions $X(0)=$ $\dot{X}(0)=0$. If the magnetic force $F$ is turned on at the time $t=0$, analogous conditions take place for the position $x^{*}$. Then the full MSD in the laboratory frame will be

$$
X_{t}(t)=\left\langle\left[x_{t}(t)-x(0)+x^{*}(t)-x^{*}(0)\right]^{2}\right\rangle=X(t)+x^{* 2} .
$$

Since $\langle x(t)\rangle=\left\langle x(t)-x^{*}(t)\right\rangle=0$, we have $\left\langle\dot{x}_{t}\right\rangle=\dot{x}^{*}$, then $\langle\dot{\phi}\rangle=-\alpha \dot{x}^{*} / \lambda-2 \omega_{\text {pin }} x^{*} / \lambda+g \mu_{B} B / \hbar$ and the averaged induced current is calculated from (1) as

$$
\begin{aligned}
& \langle I(t)\rangle=C\left[\langle\dot{\phi}(t)\rangle-\beta \frac{\dot{x}^{*}}{\lambda}\right]= \\
& \quad C\left[\frac{g \mu_{B} B}{\hbar}-(\alpha+\beta) \frac{\dot{x}^{*}}{\lambda}-2 \omega_{\text {pin }} \frac{x^{*}}{\lambda}\right],
\end{aligned}
$$

Now the problem is fully formulated and what we need is just to complement the obtained Eqs. (20) and (21) with the solutions of (18) and (6).

The full MSD, calculated from (20), includes the influence of the external magnetic field

$$
\begin{gathered}
\frac{m \omega_{0}^{2}}{2 k_{B} T} X_{t}(t)=\xi(t)\left(1+\frac{N_{w}}{1+\alpha^{2}} \frac{\left(g \mu_{B} B\right)^{2}}{2 \hbar \omega_{\mathrm{pin}} k_{B} T} \xi(t)\right) \\
\xi(t)=1-\exp \left(\frac{-\gamma t}{2 m}\right)\left(\cos \omega_{1} t+\frac{\gamma \sin \omega_{1} t}{2 m \omega_{1}}\right)
\end{gathered}
$$

In the numerical calculations (Fig. 4) we used for $B$ the Walker-breakdown field $B_{w}=\alpha K / g \mu_{B}$ [4] and the temperature $T=333 \mathrm{~K}$.

The basic formula for the mean electric current $\langle I(t)\rangle$ induced by the translating and oscillating DW is (21). It contains the DW position function $\dot{x}^{*}(t)$, calculated in the same way as $X(t)$ from (7), with only the change of the constant multiplier on the right to $F /\left(m \omega_{0}^{2}\right)$

$$
x^{*}(t)=\frac{F}{m \omega_{0}^{2}}\left[1-\exp \left(\frac{-\gamma t}{2 m}\right)\left(\cos \omega_{1} t+\frac{\gamma \sin \omega_{1} t}{2 m \omega_{1}}\right)\right] .
$$


Using (21), we find

$$
\begin{aligned}
& \frac{\langle I(t)\rangle}{\langle I(0)\rangle}=\exp \left(-\frac{\gamma t}{2 m}\right)\left\{\cos \omega_{1} t+\right. \\
& \left.\quad \frac{\sin \omega_{1} t}{2}\left[\frac{\gamma}{m \omega_{1}}-(\alpha+\beta) \frac{\omega_{1}}{\omega_{p i n}}\left(1+\frac{\gamma^{2}}{4 m^{2} \omega_{1}^{2}}\right)\right]\right\}
\end{aligned}
$$

Due to the time dependence of the mean position (23), this result significantly differs from that found in [4], where the averaged current is time-independent. The solution (24) is represented in Fig. 5 for the same parameters as in the previous graphs.

\section{CONCLUSION}

The present contribution comes from two recent achievements in the experimental and theoretical study of magnetic domain walls. First, coming from the representation of the DW as an object with particle-like properties, in the experiment [2] the mass of the DW has been determined. As to the theory, in the works by Lucassen, Duine and coauthors (see [4] and references therein) the thermal fluctuations of DWs were thoroughly described. We have joined these two approaches and considered the DW dynamics in a wire as a motion of a Brownian particle subjected to thermal noise. The studied DW is driven by the random Langevin force, damped by a Stokes-like friction force linearly depending on the velocity, and pinned by irregularities in the material. Using the theory [4], the Langevin equation for such a particle has been obtained in the linear approximation. Due to the pinning the DW oscillates in a harmonic potential and in the presence of an external magnetic field this motion is driven by a constant force. The resulting stochastic equation has the same form as for a noisy harmonic oscillator. We have solved it by separating the DW motion in a deterministic part and a stochastic contribution to the displacement. The method of finding the stochastic contribution was borrowed from the statistical theory of the Brownian motion of particles. We believe that its efficiency was clearly demonstrated and the used approach could be useful in a number of related problems, not only in the dynamics of domain walls. By this way we calculated the mean position of the DW and the time correlation functions for the DW coordinate and velocity. In addition, the spectral densities of these functions and the mean electric current induced in the wire by the moving DW have been calculated. The obtained results notably differ from the previous calculations [4]. The main difference is in the time dependence of the induced current, which is not constant, as calculated in [4]. It shows a damped oscillatory behaviour and only for $t \rightarrow \infty$ approaches a constant value. The presented results are easy to verify theoretically and they could be examined experimentally. It should be however noted that they are valid only for rigid DWs described by small chiralities $\phi$. In the opposite case the nonlinear nature of the equations governing the DW motion makes our approach to the solution of the Langevin equation inapplicable. One possibility towards a progress in this class of problems is based on the Fokker-Planck equation for the probability density of the variables describing the DW motion. Currently, our attempts are oriented in this direction.

\section{Acknowledgement}

This work was supported by the Agency for the Structural Funds of the EU within the project NFP 26220120021, and by the grant VEGA 1/0300/09.

\section{REFERENCES}

[1] TATARA, G.-KOHNO, H.-SHIBATA, J. : Microscopic Approach to Current-Driven Domain Wall Dynamics, arXiv:0807. $2894 \mathrm{v} 2$ [cond-mat.mes-hall] (2008) - an upgraded version of the paper published in Phys. Rep. (2008), 468, 213-301.

[2] SAITOH, E.-MIYAJIMA, H.-YAMAOKA, T.-TATARA, G. : Current-Induced Resonance and Mass Determination of a Single Magnetic Domain Wall, Nature 432 (2004), 203-206.

[3] DÖRING, V. W. : Über die Trägheit der Wände zwischen Weisschen Bezirken, Z. Naturforsch. No. 3a (1948), 373-379.

[4] LUCASSEN, M. E.-DUINE, R. A.: Spin Motive Forces and Current Fluctuations due to Brownian Motion of Domain Walls, arXiv:0910.063v1 [cond-mat.mes-hall] (2009), submitted to "Special issue: Caloritronics in Solid State Communications..

[5] MAZO, R. M.: Brownian Motion. Fluctuations, Dynamics, and Applications, Oxford University Press, New York, 2009.

[6] DUINE, R. A.: Spin Pumping by a Field-Driven Domain Wall, arXiv:0706.3160v3 [cond- mat.mes-hall], Phys. Rev. B 77 (2008), 01440.

[7] DUINE, R. A.: Effects of Non-Adiabaticity on the Voltage Generated by a Moving Domain Wall, arXiv:0809.2201v1 [cond-mat.mes.-hall], Phys. Rev. B 79 (2009), 14407.

[8] LANDAU, L. D.-LIFSHITZ, E. M. : Statistical Physics, Part I, Nauka, Moscow, 1976, English translation: Reed Educational and Professional Publ., Oxford e.a., $3^{r d}$. Ed., 2000. (in Russian)

[9] GitTerman, M. : The Noisy Oscillator, World Scientific, Singapore, 2005.

10] COFFEY, W. T.-KALMYKOV, Yu.P.-WALDRON, J. T. : The Langevin Equation. With Applications to Stochastic Problems in Physics, Chemistry and Electrical Engineering, 2 nd Ed., World Scientific, New Jersey e.a., 2005.

11] VAN KAMPEN, N. G.: Fluctuations in nonlinear systems, Chapter 5 of Fluctuation Phenomena in Solids (BURGESS,R.E., ed.), Academic Press, New York, 1965, pp. 139-177.

[12] RISKEN, H.: The Fokker-Planck Equation. Methods of Solution and Applications, 2 nd Ed., Springer-Verlag, Berlin e.a., 1989.

13] VLADIMIRSKY, V. V.: To the Question of the Evaluation of Mean Products of Two Quantities Related to Different Moments of Time in Statistical Mechanics, Zhur. Eksper. Teor. Fiz. 12 (1942), 199-202. (in Russian)

Received 30 June 2010

Lukáš Glod, Gabriela Vasziová, Jana Tóthová and Vladimír Lisý. For biographies see No.4/2010 of this Journal.

$* * *$

This work was presented at 16-th International Conference Applied Physics of Condensed Matter, June 16-18, 2010, Malá Lučivná, Slovakia. 